\title{
Dynamics Simulation of Conducting Polymer Interchain Interaction Effects on Polaron Transition
}

\author{
José Rildo de Oliveira Queiroz and Geraldo Magela e Silva \\ Institute of Physics \\ University of Brasília, 70.917-970, \\ Brasília, Distrito Federal, Brazil \\ \{magela, rildo\}@fis.unb.br \\ http://www.fis.unb.br
}

\begin{abstract}
Effects of interchain interaction on the polaron-bipolaron transition on conjugated polymer are investigated. We use the Su-Schrieffer-Heeger model combined with the Pariser-Parr-Pople model modified to include interchain interaction, and an external electric field. We study the dynamics within the Time-Dependent Unrestricted HartreeFock approximation. We find that removing an electron from interacting conducting polymer chains bearing a single positively charged polaron leads to the direct transition of polaron to bipolaron state. The transition which is produced is single-polaron to bipolaron transition whose excitation spectrum explains the experimental data. We also find that depending on how fast the electron is removed, a structure that contains a bipolaron coupled to a breather is created.
\end{abstract}

Keywords: Polaron, Dynamics, Interchain-Interaction, Transition.

\section{Introduction}

Properties of organic light-emitting diodes, transistors and lasers are due to conjugated polymers. 112 Their semiconductor properties are related to the nonlinear electronic response of the coupled electron-lattice system. 3] These nondegenerate ground state $\pi$-electron materials are able to form, by the electronlattice interaction, self localized electron states called polaron and bipolaron. Bipolarons and polarons are though to play the leading role in determining the charge injection, optical and transport properties of conducting polymers. 4. Bipolarons and polarons are self-localized particle-like defects associated with characteristic distortions of the polymer backbone and with quantum states deep in the energy gap due to strong electron-lattice coupling. A polaron has a spin $\pm 1 / 2$ and an electric charge $\pm e$, whereas a bipolaron is spinless with a charge $\pm 2 e$.

A critical problem in the understanding of these materials is the consistent description of the dynamics of mechanism of creation, stability and transition of polarons to bipolarons. 
UV-Vis-NIR spectroscopy studies on poly(p-phenylene vinylene) combined to the follow-up of the kinetics of doping with iodine vapor were reported and interpreted as direct observations of the formation of polaronic charge carriers. 1] However, by following different doping levels with $I_{2}$ doping, bipolaron formation is identified as well showing that polarons and bipolarons coexist in the oxidized polymer. These results corroborate the findings of Steinmüller et al [5] where the evolution of the gap states of bithiophene as a model system for polythiophene for different n-doping levels was followed by ultraviolet photo-emission spectroscopy (UPS) and electron-energy-loss spectroscopy (EELS).

The polaron-bipolaron transition problem was explicitly addressed by Cik et al in poly(3-dodecyl thiophene) in connection with temperature changes. 6] They found that when the sample was heated and subsequently cooled, there was an amplification of the diamagnetic inter- and intra-chain bipolarons. Kaufman et al study of polypirrole 7] by optical-absorption spectroscopy and ESR also pointed that the metastable states possess spin, while the stable states do not.

Many efforts have been devoted to describe the polaron-bipolaron conundrum theoretically. Electronic structure calculations, 8 extensions of the Su-SchriefferHeeger model, 910, the Pariser-Parr-Pople model, 11] as well as combinations of them 12 have been used to determine the relative prevalence of each excited state in various regimes. Several different approaches 912 1314 point to bipolaron system been more stable than the polaron system when dopants are taken into account.

Two mechanisms have been put forward to explain the transition from polaron to bipolaron states. Polarons recombination into bipolaron, 6 7 715] where the bipolaron is generated when polarons with the same electric charge meet each other; and single-polaron to bipolaron transition, 11316] where the polaron structure is transformed by the addition of one extra charge.

Here, we report the results of dynamical calculations on polaron-bipolaron transition mechanism with interacting chains. We use the Su-Schrieffer-Heeger model[17] modified to include the Coulomb interaction via extended Hubbard model, Brazovskii-Kirova (BK) symmetry breaking terms, the action of an external electric field, and interchain interactions. 12. The time-dependent equations of motion for the lattice sites and the $\pi$-electrons are numerically integrated within the Time-Dependent Hartree-Fock approximation.

Stafström et al have used a similar approach to treat polaron migration between chains (ref. [20]). Nevertheless, they did not consider electron Coulomb interaction and symmetry breaking terms. Furthermore, open end boundary conditions were used.

In agreement with UV-Vis-NIR spectroscopy, [1] UPS and EELS measurements, 5. our theoretical studies of the transition indicate that the single-polaron to bipolaron transition is the preferred mechanism of polaron-bipolaron transition in conjugated polymers.

We find that a breather mode of oscillation is created at the lattice in connection with the transition around the bipolaron. The breather amplitude is 
associated with how fast the extra charge is added to the system. Moreover, the created bipolaron is trapped by the breather.

\section{$2 \quad$ Model}

A SSH-Extended Hubbard type Hamiltonian modified to include an external electric field and interchain interaction is considered. The Hamiltonian is given by:

$$
H=H_{1}+H_{2}+H_{i n t}
$$

where,

$$
\begin{aligned}
H_{j} & =-\sum_{i, s}\left(t_{j_{i, i+1}} C_{j_{i+1, s}}^{\dagger} C_{j_{i, s}}+H . c\right) \\
& +U \sum_{i}\left(n_{j_{i \uparrow}}-\frac{1}{2}\right)\left(n_{j_{i \downarrow}}-\frac{1}{2}\right) \\
& +V \sum_{i}\left(n_{j_{i}}-1\right)\left(n_{j_{i+1}}-1\right) \\
& +\sum_{i} \frac{K}{2} y_{j_{i}}^{2}+\sum_{i} \frac{M}{2} \dot{u}_{j_{i}}^{2}, \quad j=1,2
\end{aligned}
$$

and

$$
\begin{aligned}
H_{i n t}= & -\sum_{i=p, s}^{q} t_{\perp}\left(C_{1_{i, s}}^{\dagger} C_{2_{i, s}}+C_{2_{i, s}}^{\dagger} C_{1_{i, s}}\right) \\
& +\sum_{s} V_{p}\left(C_{1_{m, s}}^{\dagger} C_{1_{m, s}}+C_{1_{m+1, s}^{\dagger}}^{\dagger} C_{1_{m+1, s}}\right)
\end{aligned}
$$

$C_{i, s}^{\dagger}\left(C_{i, s}\right)$ is the creation (annihilation) operator of a $\pi$ electron with spin $s$ at the $i$ th lattice site, $n_{i, s} \equiv C_{i, s}^{\dagger} C_{i, s}$ is the number operator, and $n_{i}=\sum_{s} n_{i, s}$. $y_{n} \equiv u_{n+1}-u_{n}$, where $u_{n}$ is the displacement of $n$th CH-group from equilibrium position in the undimerized phase. $t_{j_{n, n+1}}=\exp (-i \gamma A)\left[\left(1+(-1)^{n} \delta_{0}\right) t_{0}-\alpha y_{j_{n}}\right]$, $t_{0}$ is the transfer integral between the nearest neighbor sites in the undimerized chains, $t_{\perp}$ is the hopping integral between sites with the same index on different chains from $p$ site to $q$ site, $\alpha$ is the electron-phonon coupling, $\delta_{0}$ is the BK symmetry-breaking parameter. $M$ is the mass of a $\mathrm{CH}$ group, $K$ is the spring constant of a $\sigma$-bond, $U$ and $V$ the on-site and nearest-neighbor Coulomb repulsion strengths, respectively. $\gamma \equiv e a /(\hbar c), e$ is the absolute value of the electronic charge, $a$ the lattice constant, and $c$ the light velocity. The relation between the time-dependent vector potential $A$ and the uniform electric field $E$ is given by $E=-\frac{1}{c} \dot{A}$. We use as parameters the commonly accepted values for conjugated polymers: $t_{0}=2.5 \mathrm{eV}, t_{\perp}=0.075 \mathrm{eV}, K=21 \mathrm{eV} \AA^{-2}, \alpha=4.1 \mathrm{eV} \AA^{-1}, U=0$ to $1.8 t_{0}, V=U / 2, a=1.22 \AA, \delta_{0}=0.05 t_{0}, V_{p}=0.2 \mathrm{eV}$, and a bare optical phonon energy $\hbar \omega_{Q}=\hbar \sqrt{4 K / M}=0.16 \mathrm{eV} \cdot[19]$ 
The dynamics of the lattice part is made with the Euler-Lagrange equations and the Schrödinger $\pi$-electrons equation of motion is solved within the unrestricted time-dependent Hartree-Fock approximation. It should be pointed out that both equations depend explicitly on the occupation number of the oneparticle electronic states. 12

In order to perform the dynamics, an initial self-consistent state is prepared solving the equations of motion for the lattice and $\pi$-electrons simultaneously. [20] Periodic boundary conditions are considered. The initial state is taken in equilibrium $(E=0)$. Therefore, we have $\dot{u}_{n}=0$ for all $n$ in the initial state.

The equations of motion are solved by discretizing the time variable with a step $\Delta t$. The time step $\Delta t$ is chosen so that the change of $u_{i}(t)$ and $A(t)$ during this interval is always very small in the electronic scale. 12 .

\section{Simulation Results}

One more hole is injected in polymer chains bearing already positively charged polarons. Since charged excitations defects can be created by quite different means: photoexcitations, chemical doping or direct charge injection via electronic device, we performed simulations where the extra electron is taken from the system during different time intervals $(\Delta T)$. We varied $\Delta T$ from 0 to $100 \mathrm{fs}$. The shorter time intervals simulate photoexcitations and the direct charge injection. The longer time intervals account for the different impurity addition procedures associate with chemical doping. The electron is taken from the highest occupied level using the following expression

$$
O_{F}(t)=\frac{1}{2}\left[1+\cos \left(\frac{\pi\left(t-t_{i}\right)}{\Delta T}\right)\right]
$$

for $t$ between $t_{i}$ and $t_{i}+\Delta T$. Here, $t_{i}$ is the time when the hole injection begins and $O_{F}(t)$ is the occupation number of the Fermi level.

We have considered two polymeric interacting chains with $N=60$ sites each, containing initially two positively charged polaron in all simulations. We use a mean charge density $\bar{\rho}_{i}(t)$, derived from the charge density $\rho_{i}(t)$, and the order parameter $\bar{y}_{i}(t)\left[y_{i}(t)=u_{i+1}(t)-u_{i}(t)\right]$ to analyze the simulations. 19. The dynamics of the system is followed during 100,000 time steps spanning $400 \mathrm{fs}$.

A smooth transition of one of the polarons to a bipolaron, in its respective chain, is obtained after the adiabatic removal $(\Delta T>80 \mathrm{fs})$ of the third electron. Figure 1 shows the time evolution of the energy levels neighboring and inside the energy gap. It can be seen that the energy levels associated with the polaron move in the middle-gap direction assuming a bipolaron conformation. The small oscillation of the levels are due to lattice oscillations induced by the hole injection perturbation.

Figure 2 presents bond length order parameter of chains 1 and 2 . It should be noted that we use periodic boundary conditions, therefore, the order parameter of chain 1 (Fig. 2(a)) represents a polaron around site 1 (it begins at site 45, it goes until site 60 and it continues from site 1 to site 15). Positively charged 


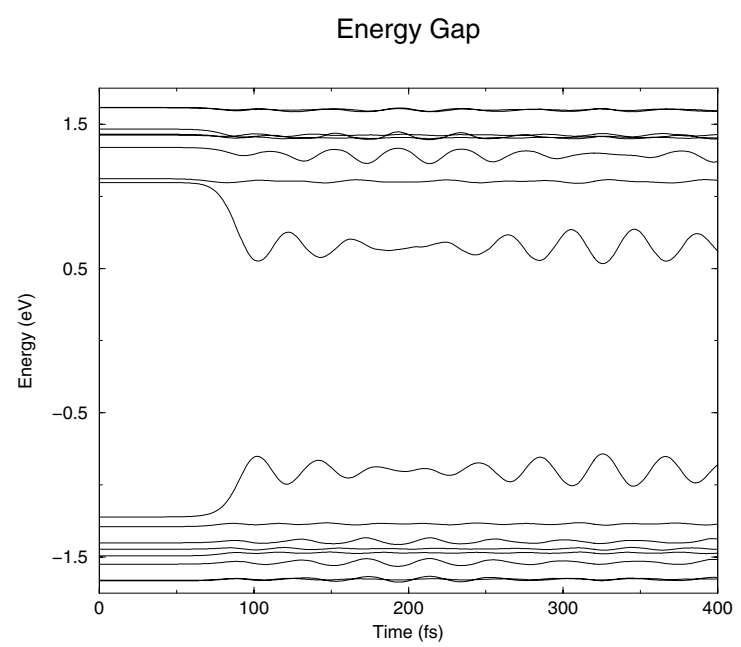

Fig. 1. Time evolution of energy levels inside and around the gap in an adiabatic transition. The spin up levels are shown. The system changes from polaron levels $(t<$ $80 \mathrm{fs})$ to bipolaron levels configuration $(t>100 \mathrm{fs})$.

polarons repel each other. They stay apart from each other as far as possible. The polaron-bipolaron transition occurs in chain 2. This clear transition happens in chain 2 as an apparent spontaneous symmetry breaking. Nevertheless, the presence of an impurity on chain 2 leads to a symmetry breaking and the association of one polaron to it. It is obtained that the polaron associated with the impurity makes the transition to bipolaron.

Effects of interchain interaction were addressed by varying the extent of the interacting region ( $p$ and $q$ in the Hamiltonian). For the transitions where two chains interact only on half of their length ( $p=31$ and $q=60)$, one polaron stays in the interacting region and the other stays in the non-interacting region due again to Coulomb repulsion. It is obtained that the polaron-bipolaron transition happens with the polaron in the interacting region. Therefore, the interchain interaction is also effective in promoting the transition.

Figure 3 presents a very special case where two polarons merge to create a bipolaron. This case is quite the originally suggested process for the polaronbipolaron transition. 21] Here, after the hole injection, there appears an exciton lasting for about $200 \mathrm{fs}$ and then the bipolaron takes place in the lattice. Nevertheless, it should be noted that this happens when one chain has a high density of polarons and the other one has initially none of them. It can be clearly seen that two polarons in chain 1 merges to a single bipolaron and another polaron appears in chain 2 due to interchain interaction and Coulomb repulsion.

The fast removal of the third electron $(\Delta T<80 \mathrm{fs})$ leads to the appearance of a breather oscillation mode in the lattice. This breather appears at the bipolaron position. As a matter of fact, the bipolaron is trapped by the breather. 19] 


\section{Polarons on Neighboring Chains}

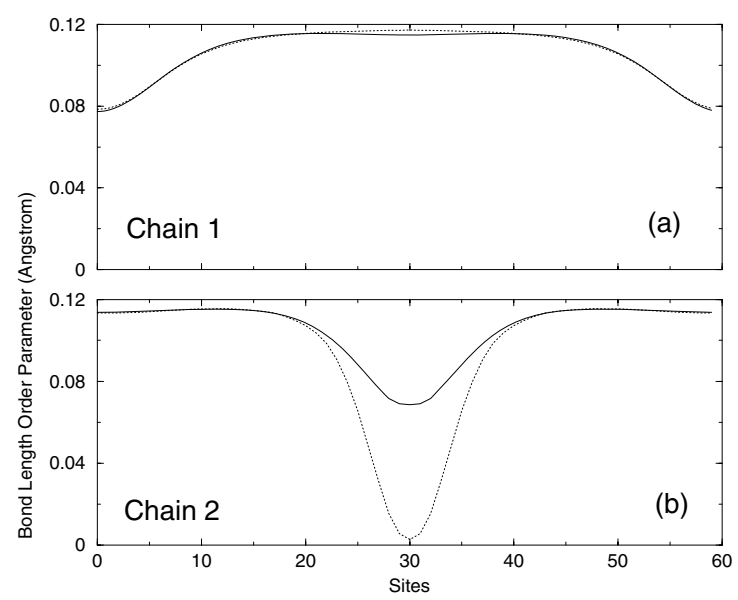

Fig. 2. Evolution of the bond length order parameter of two neighboring chains. Solid line: initial configuration; dotted line: final configuration. The polaron on chain 1 (Fig. $2(\mathrm{a})$ ) remains stable after the extra electron is adiabatically taken from the system, but the polaron on chain 2 (Fig. 2(b)) makes a transition and becomes a bipolaron.

\section{Polarons on the Same Chain}

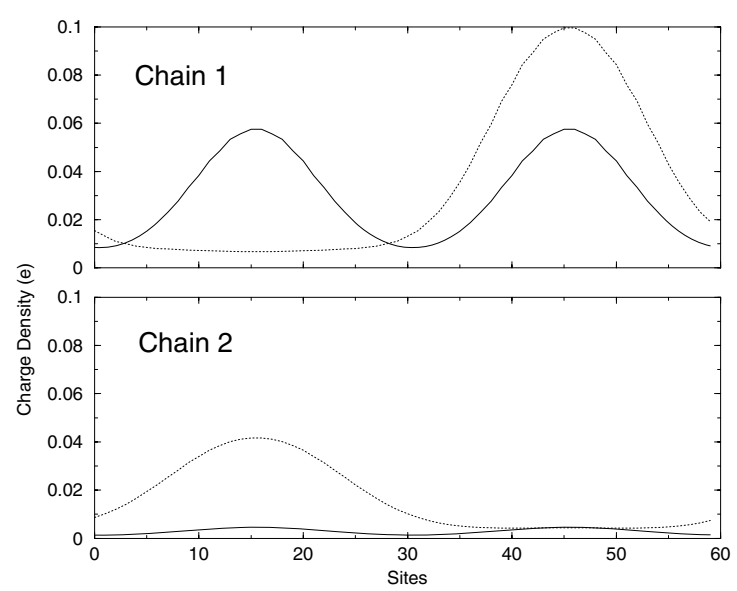

Fig. 3. Charge density of chains corresponding to simulation of Fig. 4. The initial polarons on chain 1 (solid line) coalesce on a bipolaron (dotted line). There is also the creation of a polaron on chain 2 (dotted line).

This trapping of the Bipolaron leads to a reduction of the Bipolaron mobility. Furthermore, the breather oscillation frequency could be detected by infrared 
spectroscopy and its presence in association with bipolarons should have effects on the overall conduction properties of the material.

The distinction between adiabatic and non-adiabatic injection effects is thought to be associated to the relaxation processes involved at the electronic level.

\section{Conclusions}

Effects of interchain interaction on the transition of polarons to bipolarons on two interacting conjugated polymeric chains were investigated. This study was carried out through numerical calculations.

It should be noted that our purpose is a qualitative description of the transition process on conducting polymers in general. The adopted parameter values are most that of polyacetylene because they are well known values. There was no attempt to fit the parameters to any specific polymer. Nevertheless, there was a remarkable accordance between the experimental values of the subgap transitions obtained in reference [1] for poly (p-phenine vinylene) doped with $I_{2}$, $\mathrm{FeCl}_{3}$ and $\mathrm{H}_{2} \mathrm{SO}_{4}$ and our results. There, for example, the major subgap energy differences for polarons were $1.81 \mathrm{eV}$ for $\mathrm{I}_{2} ; 2.07 \mathrm{eV}$ for $\mathrm{H}_{2} \mathrm{SO}_{4}$; and $2.08 \mathrm{eV}$ for $\mathrm{FeCl}_{3}$, whereas the same energy differences in our simulations varied from 1.93 to $2.12 \mathrm{eV}$.

We present theoretical results pointing to direct single-polaron to bipolaron transition as the favored mechanism of bipolaron formation. This result is in accordance with previous results on polaron and bipolaron dynamics calculations 12 where a pair of polarons do not spontaneously merged to produce a bipolaron.

Since we considered different characteristic time intervals for the hole insertion in the chain, in order to simulate different ways of bipolaron generation (photoproduction, chemical doping or direct charge injection), we obtained different responses from the lattice. It is found that the non-adiabatic electron removal led to the formation of an associated breather oscillation mode in the chain. Moreover, the breather interacts with the newly formed bipolaron trapping it around its position. The trapping and depinning of bipolarons from breathers have direct influence on the mobility of that charge carrier in the chain.

Based in our numerical simulations, we suggested two experimental approaches to better understand the polaron-bipolaron transition mechanism. First, the verification of the presence and quantity of breathers associated with different transition regimes. Second, the change in bipolarons mobility due to the trapping effect of breathers.

Acknowledgments. We thank CAPES, FINATEC and CNPQ for financial support. 


\section{References}

1. Fernandes, M. R., Garcia, J. R., Schultz, M. S., and Nart, F. C.: Polaron and bipolaron transitions in doped poly(p-phenylene vinylene) films. Thin Sol. Films 474 (2005) 279.

2. Burroughes, J., Bradley, D. D. C., Brown, A. R., Marks, R. N., Mackay, K., Friend, R. H., Burn, P. L., and Holmes, A. B.: Light-emitting diodes based on conjugated polymers. Nature 347 (1990) 539.

3. Jeckelmann, E., and Baeriswyl, D.: The metal-insulator transition in polyacetylene: variational study of the Peierls-Hubbard model. Synth. Met. 65 (1994) 211.

4. Furukawa, Y.: in Primary Photoexcitations in Conjugated Polymers, edited by N. S. Sariciftci (World Scientific, Singapore, 1997) 496.

5. Steinmüller, D., Ramsey, M. G., and Netzer, F. P.: Polaron and bipolaronlike states in n-doped bithiophene. Phys. Rev. B 47 (1993) 13323.

6. Cik, G., Sersen, F., and Dlhan, L. D.: Thermally induced transitions of polarons to bipolarons in poly(3-dodecylthiophene). Synth. Met. 151 (2005) 124.

7. Kaufman, J. H., and Colaneri, N.: Evolution of Polaron States into Bipolarons in Polypyrrole. Phys. Rev. Lett. 53 (1984) 1005.

8. Geskin, V. M., and Bredas, J. -L.: Polaron Pair versus Bipolaron on Oligothiophene Chains: A Theoretical Study of the Singlet and Triplet States. ChemPhysChem 4 (2003) 498.

9. Saxena, A., Brazovskii, S., Kirova, N., Yu, Z. G., and Bishop, A. R.: Stability of bipolarons in conjugated polymers. Synth. Met. 101 (1999) 325.

10. Xie, S-J., and Mei, L-M.: Transition between bipolaron and polaron states in doped heterocycle polymers. Phys. Rev. B 50 (1994) 13364.

11. Yao, K. L., Han, S. E., and Zhao, L.: The polaron and bipolaron states of poly(phenylene vinylene). J. Chem. Phys. 114 (2001) 6437.

12. e Silva, G. M.: Electric-field effects on the competition between polarons and bipolarons in conjugated polymers. Phys. Rev. B 61 (2000) 10777.

13. Irle, S., and Lischka, H.: Combined ab initio and density functional study on polaron to bipolaron transitions in oligophenyls and oligothiophenes. J. Chem. Phys. 107 (1997) 3021.

14. Bredas, J. L., Scott, J. C., Yakushi, K., and Street, G. B.: Polarons and Bipolarons in polypyrrole: Evolution of the band structure and optical spectrum upon doping. Phys. Rev. B 30 (1984) 1023.

15. Farias, G. A., da Costa, W. B., and Peeters, F. M.: Acoustical polarons and bipolarons in two dimensions. Phys. Rev. B 54 (1996) 12835.

16. Verbist, G., Peeters, F. M., and Devreese, J. T.: Large bipolarons in two and three dimensions. Phys. Rev. B 43 (1991) 2712.

17. Su, W. P., Schhrieffer, J. R., and Heeger, A. J.: Soliton excitations in polyacetylene. Phys. Rev. B 22 (1980) 2099; 28 (1983) 1138.

18. Johansson, A., and Stafström, S.: Polaron Dynamics in a System of Coupled Conjugated Polymer Chains. Phys. Rev. Lett 86 (2001) 3602.

19. Lima, M. P., and e Silva, G. M.: Dynamical evolution of polaron to bipolaron in conjugated polymers. Phys. Rev. B 74 (2006) 224304.

20. Pinheiro, C. da S., and e Silva, G. M.: Use of polarons and bipolarons in logical switches based on conjugated polymers. Phys. Rev. B 65 (2002) 94304.

21. Moses, D., Wang, J., Yu, G., and Heeger, A. J.: Temperature-Independent Photoconductivity in Thin Films of Semiconducting Polymers: Photocarrier Sweep-Out Prior to Deep Trapping. Phys. Rev. Lett 80 (1998) 2685. 